\title{
EXOhSPEC collimator mechanical design
}

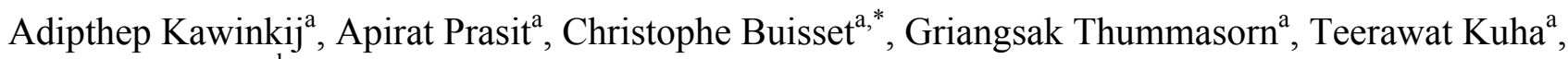
Esther Lhospice $^{\mathrm{a}, \mathrm{b}}$, Hugh R.A. Jones ${ }^{\mathrm{c}}$, William E. Martin ${ }^{\mathrm{c}}$, Ronny Errmann ${ }^{\mathrm{c}}$, Piyamas Choochalerm ${ }^{\mathrm{c}}$, Guillem Anglada-Escude ${ }^{\mathrm{d}}$, David Campbell ${ }^{\mathrm{c}}$, Clark Baker ${ }^{\mathrm{c}}$, Somsawat Rattanasoon ${ }^{\mathrm{a}}$, Suparerk Aukkaravittayapun $^{\mathrm{a}}$, Thirasak Panyaphirawat ${ }^{\mathrm{a}}$, Apichat Leckngam ${ }^{\mathrm{a}}$, David Mkrtichian ${ }^{\mathrm{a}}$, Saran Poshyachinda ${ }^{\mathrm{a}}$, Boonrucksar Soonthornthum ${ }^{\mathrm{a}}$

${ }^{a}$ National Astronomical Research Institute of Thailand (NARIT), 260 Moo 4, T. Donkaew, A. Maerim, Chiangmai, 50180, Thailand; ${ }^{b}$ Institut d'optique Graduate School, 2 Avenue Augustin Fresnel, 91120 Palaiseau, France; ${ }^{\mathrm{c} C e n t r e}$ for Astrophysics Research, University of Hertfordshire, Hatfield, AL10 9AB, UK; ' Queen Mary University of London, Mile End Road, London E1 4NS

\begin{abstract}
The Exoplanet High-Resolution Spectrograph (EXOhSPEC) is a high-resolution spectrograph for the characterisation of exoplanets with the Thai National Telescope. The folded version of this instrument comprises one triplet lens to collimate the beam incident on the grating and to focus the beam reflected by the grating onto the camera. This collimator comprises three lenses L1, L2 and L3 of diameter varying between $50 \mathrm{~mm}$ and $60 \mathrm{~mm}$. We specified the barrel to guarantee a maximum decenter of the lenses equal to $25 \mu \mathrm{m}$. The maximum error in the orientation of each single lens is specified to be lower than $0.03^{\circ}$. The proposed concept is based on a semi-kinematic mounting which is used to restrain these lenses with 6 and $30 \mathrm{~N}$ of preloads on the axial and lateral directions to ensure their stability. These preloads are applied to the lenses using the elastic pushing force of silicone elastomers and spring force from ball-plungers. We present the design of the collimator and the assembly method. Our Finite Element Analyses show that the maximum surface error induced by the preloads is lower than $60 \mathrm{~nm}$ Peak-To-Valley on each optical surface of L1, L2, and L3. We describe our manufacturing process using NARIT's CNC machine and its validation using our Coordinate-Measuring Machine.
\end{abstract}

Keywords: Spectroscopy, opto-mechanics, design, precision engineering, astronomy.

\section{INTRODUCTION}

The Exoplanet High-resolution Spectrograph (EXOhSPEC) [1] is a high-resolution Echelle spectrograph whose objective is to provide high resolution spectra with the $2.3 \mathrm{~m}$ Thai National Telescope (TNT) [2]. The collimator of EXOhSPEC is the core part of the spectrograph and it has been designed to provide an image quality close to the diffraction limit in the visible where the spectrograph will operate. This collimator is made of an apochromatic triplet that collimates the beam coming from the fiber source output face and focuses the reflected beam from the grating on the detector. The layout of the EXOhSPEC ZEMAX model is shown in Figure 1. The triplet lenses are made of Schott N-FK51A and N-KZFS11 glasses with diameter varying between 50.0 to $60.0 \mathrm{~mm}$.

In Section 2, we describe the optical design of the EXOHSPEC achromatic triplet and discuss the requirements for the mechanical design and the manufacturing constraints. Section 3 presents the concept of the barrel that aims at positioning the lens at the correct locations by applying axial and lateral preloads. Section 4 describes this barrel and Section 5 presents our result Finite Element Analyses. We show that the maximum surface error induced by the preloads applied is equal to $\lambda / 9$ over the optical surface of the incident beam. In Section 6 , we describe the manufacturing processes used at NARIT by using a Computer Numerical Control (CNC) machine. We also show the result of the measurement of the dimensions of the as-built barrel of the collimator and that these results are compliant with the specifications. 


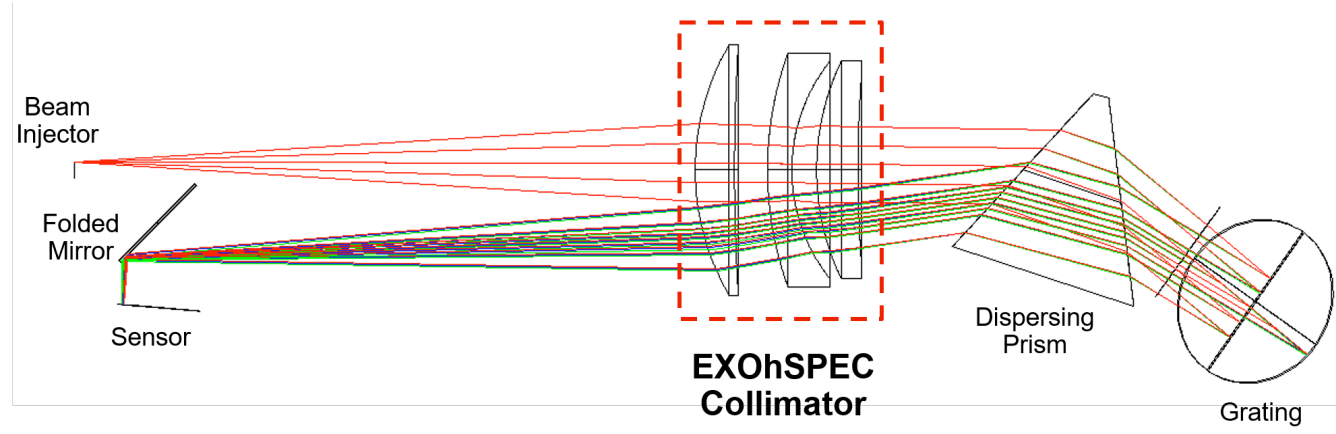

Figure 1. EXOhSPEC optical design showing the major elements and emphasizing the central role of the collimator.

\section{OPTICAL DESIGN AND REQUIREMENTS}

\subsection{Collimator optical design}

The EXOhSPEC triplet comprises three lenses L1, L2 and L3 (Figure 3) which have been optimized by using the ZEMAX software to reach diffraction limited images over the spectral interval from 400 to $700 \mathrm{~nm}$. The parameters of these lenses are represented in Table 2. The lenses L1 and L2 are made of N-FK51A while the material of the lens L3 is N-KZFS11. The mechanical diameter of the lenses L1, L2 and L3 are $60 \mathrm{~mm}, 56$ and $52 \mathrm{~mm}$ respectively. The central thickness of each lens was been adjusted during the optimization process. This thickness is equal to $9.5 \mathrm{~mm}$ for L1, $5.4 \mathrm{~mm}$ for L2 and $10 \mathrm{~mm}$ for L3. The distance between L1 and L2 is equal to $8 \mathrm{~mm}$ and $6 \mathrm{~mm}$ between L2 and L3.

Table 2. Achromatic triplet parameters for collimator lenses L1, L2 and L3.

\begin{tabular}{crrrrr}
\hline Lens & Surface & $\begin{array}{r}\text { Radius of Curvature } \\
(\mathrm{mm})\end{array}$ & $\begin{array}{r}\text { Diameter } \\
(\mathrm{mm})\end{array}$ & $\begin{array}{r}\text { Thickness } \\
(\mathrm{mm})\end{array}$ & Glass \\
\hline L1 & S1 & 58.774 & 60.00 & 9.5 & N-FK51A \\
& S2 & 474.37 & - & - & \\
L2 & S3 & 81.529 & 56.00 & 5.9 & N-KZFS11 \\
& S4 & 41.224 & - & - & \\
L3 & S5 & 59.604 & 52.00 & 10.0 & N-FK51A \\
& S6 & 287.33 & - & - & \\
\hline
\end{tabular}

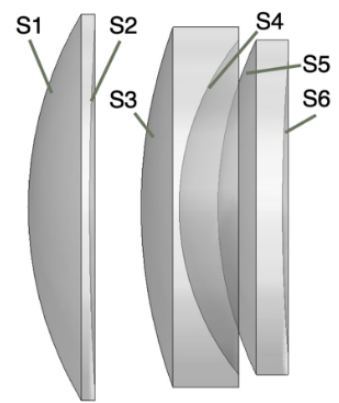

L1 L2 L3

Figure 3. Solidworks view of the achromatic triplet lenses with L1 that comprises the optical surfaces S1, S2. Then L2 that comprises the surfaces S3, S4 and L3 that comprises the optical surfaces S5 and S6. 


\subsection{Collimator barrel mechanical requirements}

The mechanical requirements of the collimator barrel are represented in Table 3 . These requirements have been derived from the optical tolerancing analyses [1] that are aimed at estimating the image quality after assembling the lenses in the barrels and aligning EXOhSPEC. The maximum difference between the real lens real position (with respect to its real orientation) and the theoretical position (with respect to its theoretical orientation) needs to be less than $25 \mu \mathrm{m}$ (with less than a $0.03^{\circ}$ tilt). It is important to mention that these tolerances are in line with NARIT manufacturing capabilities as discussed in the next section. We also require that all the internal and external parts of the collimator are black anodized. This is required in order to reduce the amount of stray light due to scattering on the collimator mechanical surfaces.

Table 3. Mechanical requirements of collimator.

\begin{tabular}{crcr}
\hline Lens & $\begin{array}{r}\text { Decenter Z } \\
(\mu \mathrm{m})\end{array}$ & $\begin{array}{r}\text { Decenter X, Y } \\
(\mu \mathrm{m})\end{array}$ & $\begin{array}{r}\text { Tilt X, Y } \\
\left({ }^{\circ}\right)\end{array}$ \\
\hline L1 & \pm 25 & \pm 25 & \pm 0.03 \\
L2 & \pm 25 & \pm 25 & \pm 0.03 \\
L3 & \pm 25 & \pm 50 & \pm 0.03 \\
\hline
\end{tabular}

\subsection{NARIT high precision workshop manufacturing and measurement capabilities}

The NARIT mechanical workshop includes the high performance Computer Numerical Control machines Haas VF-3, and VF-4 . These machines are operated for high precision manufacturing. Based on our previous experience of manufacturing mechanical parts for the TNT focal reducer [references], we assume that the maximum fabrication error induced during the process is $\pm 25 \mu \mathrm{m}$. These machining errors are assumed to be normally-distributed, and accumulated using standard root sum squared uncertainty analysis [3].

The workshop facility in NARIT also comprises one high performance Global Classic SR 05.07.05 Coordinate Measurement Machine (CMM) manufactured by the company Hexagon [4]. This CMM has been used to control the quality and verify the geometric error of the EXOhSPEC barrel. This machine includes the standard ISO10360-2 HR-S-X1C scanning probe and the PC-DMIS PRO measurement software. We estimate that the theoretical measurement accuracy is close to $\pm 2 \mu \mathrm{m}$. The measurement operation is made in a room with a temperature control from an air conditioning system. This, ensures the temperature is stable at $+/-3$ degree during the measurements.

\section{CONCEPTUAL DESIGN}

\subsection{Integration configuration}

The conceptual mechanical design of the EXOhSPEC collimator barrel is shown in Figure 5. This barrel is based on a semi-kinematic lens mounting that includes axial, and lateral preloads to keep each lens in contact with a well defined reference plane $[3,4]$. The axial preload is obtained by using one threaded retaining ring and one elastomer ring. Each retaining ring is screwed onto the threaded part of the barrel and the elastomer ring applies the axial preload force $F_{\mathrm{a}}$ to each lens. The lateral preload of each lens is obtained by using one plastic ball-plungers. Each plunger comprises one spring of preload force $F_{1}$ that pushes the lens into the lateral reference plane.

As indicated in Figure 5, the back surface S2 (and the back surface surface S4) of the lens L1 (and respectively lens L2) is put in contact with the reference surfaces of the barrel. It is important to mention that the edge distance between L2 and L3 was not large enough to insert a spacer or a retainer ring between L2 and L3. Thus, it was not possible to put the reference plane in contact with the back face S6 of L3. For this reason, the reference surface of the lens L3 is put in contact with the front surface S6. The L3 retaining ring thus applies a pressure on L3 back surface.

Each of the L1 and L2 retainer ring comprise one elastomer ring whose design has been optimized to fit the spherical surfaces S4 and S6. In the case of the lens L3, the preload is applied to the flat portion of S6 surface and the elastomer thus has a flat surface. In the lateral direction, the reference surface of each lens is made of two contact points separated by an angle equal to 60 degree positioned at the lens edges. The preloads must be at least equal to the weight of each lens with a safety factor to guarantee that the system will be stable during operational conditions. 

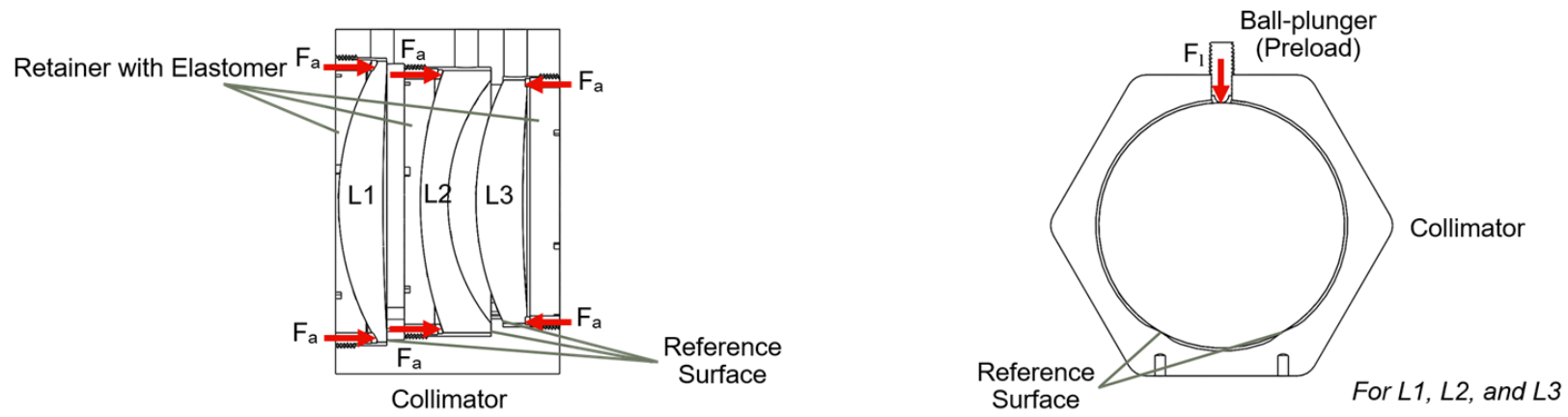

Figure 5. Conceptual design of the collimator with axial preload (left) and lateral preload (right).

\subsection{Material selection}

We selected the Aluminum alloy 6061-T6 for the collimator barrel body for the following reasons: high machinability, minimum fabrication errors and to ensure that the barrel could be black-anodized. We chose the stainless steel AISI-304 for the load-transferring components specifically (especially the fasteners). This is in order to make sure that these components will have a modulus high enough to apply the preload to the lenses and to guarantee the stability of the system. The rings are made of Silicone elastomer for the following reasons: reasonable mechanical properties, chemical inactivity, fabrication simplicity and endurance at the site of the Thailand National Observatory. We also decided to use some Polyoxymethylene polymer for each ball plunger that applies the lateral preload. This ensures that the lateral stress applied to each lens will be minimised. The list of materials used and their specifications are shown in Table 4 [5]. It is important to mention that in operational conditions, the strain will be lower than 0.1 . We deduce that i) the mechanical properties of the elastomer is constant and ii) the linear stress-strain relation can be used to calculate the preloads [reference Mechanics of materials].

Table 4. Material specifications for the barrel of the collimator.

\begin{tabular}{rrrrrrr}
\hline Materials & $\begin{array}{r}\text { Yield Strength } \\
(\mathrm{MPa})\end{array}$ & $\begin{array}{r}\text { Shear Strength } \\
(\mathrm{MPa})\end{array}$ & $\begin{array}{r}\text { Young's Modulus } \\
(\mathrm{GPa})\end{array}$ & $\begin{array}{r}\text { Shear Modulus } \\
(\mathrm{GPa})\end{array}$ & $\begin{array}{r}\text { Density } \\
\left(\mathrm{g} / \mathrm{cm}^{3}\right)\end{array}$ & $\begin{array}{r}\text { Poisson } \\
\text { Ratio }\end{array}$ \\
\hline A1 6061-T6 & 275.0 & 210.0 & 69.0 & 26.0 & 2.7 & 0.33 \\
AISI-304 & 206.8 & 400.0 & 190.0 & 75.0 & 8.0 & 0.29 \\
Polyoxymethylene & 65.5 & 55.2 & 2.8 & 1.0 & 1.4 & 0.44 \\
Silicone elastomer & 70.0 & 3.0 & $\begin{array}{r}0.001707 \\
\text { (Shore 50A) }\end{array}$ & 0.9 & 1.3 & 0.50 \\
& & & & & & \\
\hline
\end{tabular}

\subsection{Lens weight estimation}

We calculated the weight of each lens in order to estimate the force that will be applied by the axial and the lateral preloads. The glass types used in this achromatic triplet design are Schott N-FK51A for L1, and L3, and N-KZFS11 for L2. The densities of these glasses are equal to $3.675 \mathrm{~g} / \mathrm{cm}^{3}$ and $3.196 \mathrm{~g} / \mathrm{cm}^{3}$ respectively as indicated in Table 5 . By using the densities and the volumes of the lenses we calculated the weight of each individual lens. We estimated that the weight of lenses L1, L2 and L3 are 61.9, 67.1 and $59.5 \mathrm{~g}$ respectively. We deduce that the total weight of glass of this achromatic triplet is $188.6 \mathrm{~g}$. 
Table 5. Weight estimation for the lenses in the collimator.

\begin{tabular}{crrr}
\hline Lens & $\begin{array}{r}\text { Volume } \\
\left(\mathrm{cm}^{3}\right)\end{array}$ & $\begin{array}{r}\text { Density } \\
\left(\mathrm{g} / \mathrm{cm}^{3}\right)\end{array}$ & $\begin{array}{r}\text { Weight } \\
(\mathrm{g})\end{array}$ \\
\hline L1 & 16.841 & 3.675 & 61.891 \\
L2 & 20.996 & 3.196 & 67.103 \\
L3 & 16.201 & 3.675 & 59.539 \\
\hline
\end{tabular}

\section{DETAILED DESIGN}

\subsection{Overview}

The design of this collimator comprised of four steps. In first step we designed the achromatic triplet by using the ZEMAX software. In a second step, we exported the collimator optical design from ZEMAX into Solidworks. In the third step, we designed the mechanical structure of the barrel using the Solidworks software. In the fourth step, we performed Finite Element Analysis to verify that the deformation of the optical surface of each lens induced by the preloads was acceptable. Figure 8 represents the EXOhSPEC barrel semi-kinematic mounting that comprises eleven parts:

- $\quad$ The barrel provides the required reference surface for fixing the position and orientation of the lenses as specified in Section 3.

- Three retainers: provide preload necessary to fix each lens position and orientation on the axial direction. These retainers are coupled with an elastomer ring to relay the preload to the lenses.

- Three ball-plungers: provide preload necessary to fix each lens position in the lateral direction. The coil spring inside each ball-plunger exerts the required preload to the lenses.

- One front Protector: protects and safely stabilizes the retainer of lens L1.

- $\quad$ One rear Protector: protects and safely stabilizes the retainer of lens L3.

- One plunger Closure: protects and encloses the ball-plunging mechanism.

- One mounting Adapter: provides the interface for mounting on an optical bench.

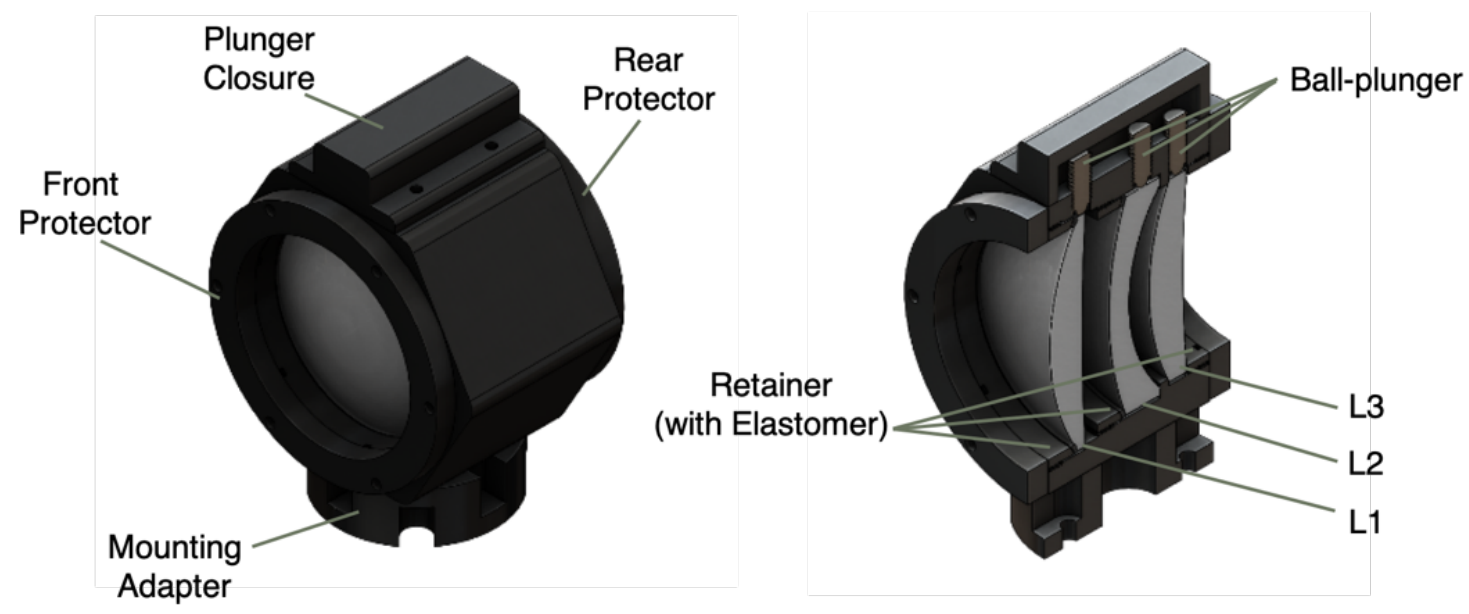

Figure 8. Overview of collimator design (left) and its cross-section view (right). 


\subsection{Axial and preload design}

We use threaded retaining rings to convert the torque applied into axial preloads to the lenses. The force applied to each lens is larger than weight of the lens so as to ensure sufficient stability during normal operation. One Standard ISO4762 M4 pitch is used as the mating thread between the barrel and the retainers for machinability, torque control and threadcrossing prevention.

The retainers have been designed specifically for each lens. In particular the contact surfaces or the L1 and L2 retainers have been chamfered to get near-ideal tangential interfaces with the lens convex curvatures, thus minimizing the contact stress. In the case of L3, the retainer is interfaced with the flat portion of the surface S6 and the retainer surface is thus flat.

Each Silicone elastomer is bonded with the retainer by using metal-plastic epoxy resin. Due to limited space around and between the lenses, the thickness of Silicone elastomer are $1.0 \mathrm{~mm}$ for L1 and L2 and $0.5 \mathrm{~mm}$ for L3 with a strain in the $5 \%-10 \%$ range. Under these conditions, the forces applied to each lenses is $F_{\mathrm{a}}=30 \mathrm{~N}$ with a safety factor equal to 45 .

We also designed some integration tools that will be used to rotate the retainer rings during the assembly process. The material of these tools is the polyoxymethylene polymer. This material has been selected for the following reasons: i) excellent machinability of this material and ii) safety since this material will not damage the optical surface in case of accidental contact between the tool and the lens during the assembly process.

The lateral preload applies a force $F_{1}=6 \mathrm{~N}$ to keep each lens in contact with the lateral reference surface with a safety factor equal to 9 . The preload exerting from the coiled spring located inside the ball-plunger is controlled by the external mechanical thread around the plunger. The ball inside the plunger is selected to be Polyoxymethylene to prevent excessive glass-to-metal contract stress.

\section{FINITE ELEMENT ANALYSIS}

We performed a preliminary Finite Element Analysis (FEA) to estimate the deformation of each optical surface in operational conditions. This analysis has been performed by using the simulation module of Solidworks. We assumed that the system is located in a standard sea-level environment with a gravity $\mathrm{g}=9.81 \mathrm{~m} / \mathrm{s}^{2}$. We also assumed that the preloads applied on each lens are $30 \mathrm{~N}$ in the axial direction and $6 \mathrm{~N}$ in the lateral direction as discussed in the previous section. We calculated the deformation of the optical surface over a circle of diameter equal to $20 \mathrm{~mm}$ centered on the axial axis of the lens. That correspond to the diameter of the footprint of the beam incident on the triplet lens surfaces. An example of the input problem to the Solidworks simulation is shown in Figure 9.

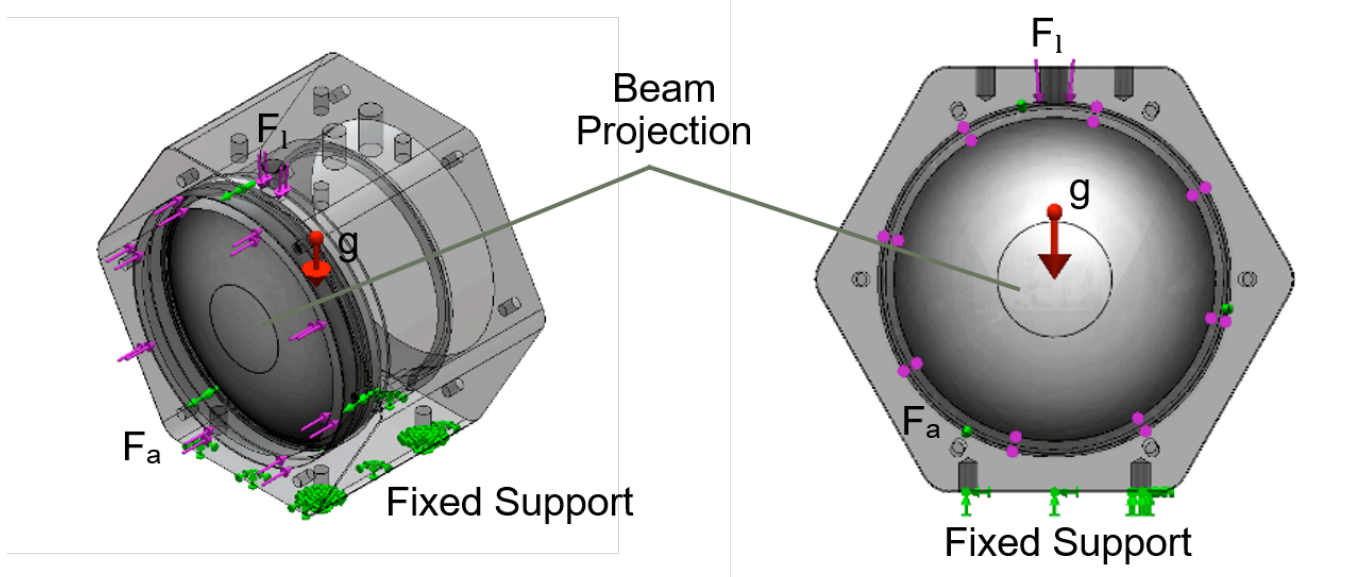

Figure 9. Input problem for L1 into the Solidworks simulation showing isometric view (left) and front view (right). 
The absolute deformations of L1, L2 and L3 optical surfaces over a circle of diameter equal to $20 \mathrm{~mm}$ are shown in the Figures 10, 11 and 12. The deformations on each surface of the lenses are summarized in Table 6. We notice that the maximum peak-to-Valley deformations of L1 optical surfaces S1 and S2 is $33 \mathrm{~nm}$ PTV. The maximum deformation of L1 surface S2 is thus $\lambda / 17$ at $500 \mathrm{~nm}$ located in the center of EXOhSPEC specified spectral range. The maximum surface errors of L2 and L3 are $22 \mathrm{~nm}$ PTV $(\lambda / 25)$ and $60 \mathrm{~nm}$ PTV $(\lambda / 9)$ respectively. The results show that the deformation induced by the preload should induce a maximum surface error of $\lambda / 9$ over the surface of the incident optical beam. These results were considered as acceptable and we thus decided to manufacture the barrel and to procure the lenses.
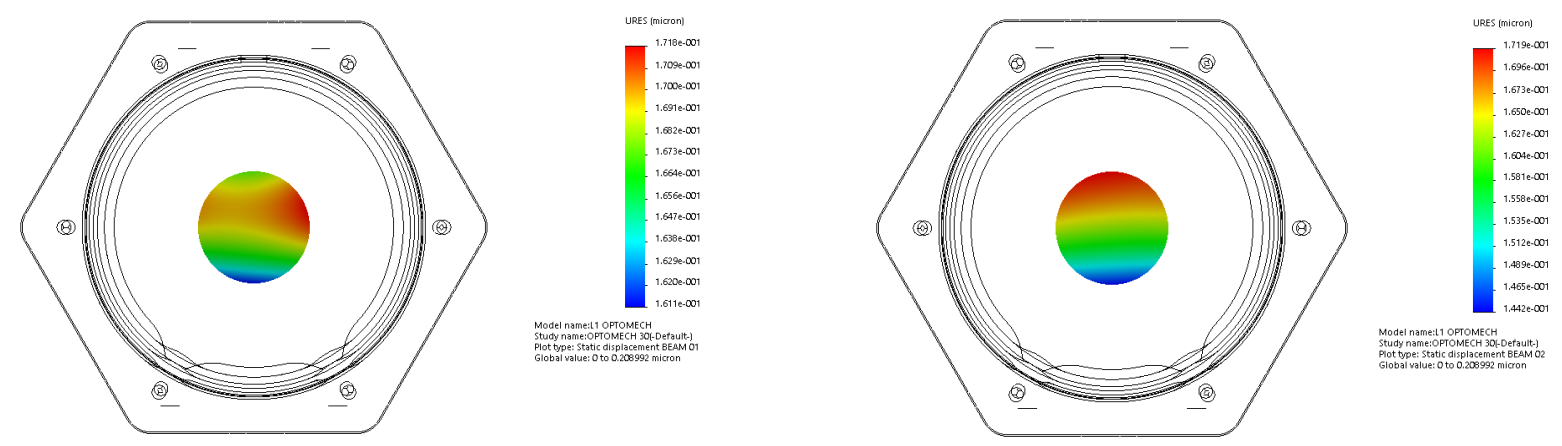

Figure 10. Deformation of L1 surface projected to the beam intersection of S1 (left) and S2 (right).
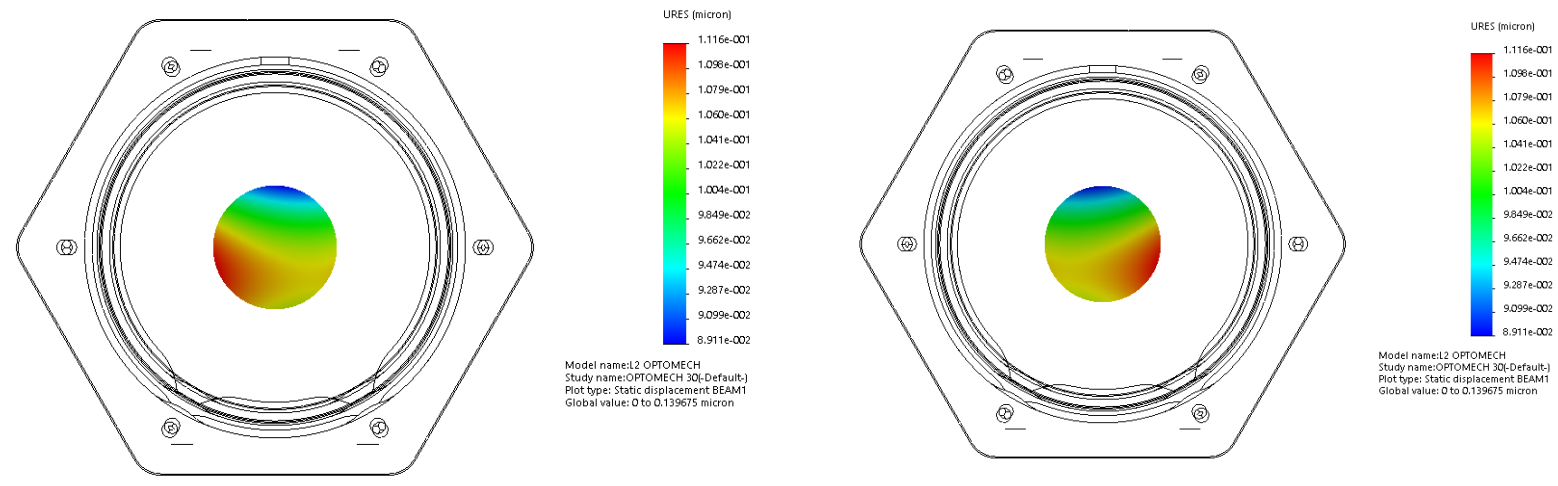

Figure 11. Deformation of L2 surface projected to the beam intersection of S3 (left) and S4 (right).
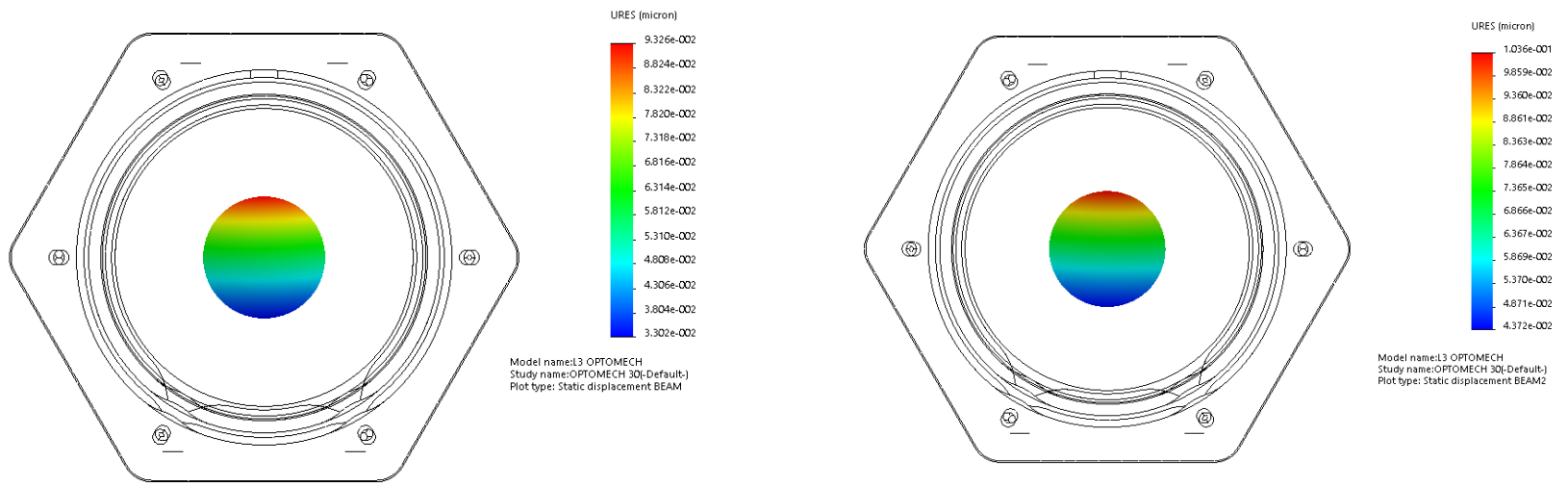
Figure 12. Deformation of L3 surface projected to the beam intersection of S5 (left) and S6 (right).

Table 6. The maximum absolute surface deformation of each lens obtained from Solidworks simulation projected on on-axis beam intersected area with $20.0 \mathrm{~mm}$ diameter.

\begin{tabular}{ccccc}
\hline Lens & Surface & $\begin{array}{c}\text { Minimum } \\
\text { Absolute } \\
\text { Deformation } \\
(\text { (min, nm) }\end{array}$ & $\begin{array}{c}\text { Maximum Absolute } \\
\text { Deformation } \\
(\text { (max, nm) }\end{array}$ & $\begin{array}{c}\text { Deformation } \\
\text { PTV } \\
(\mathrm{nm})\end{array}$ \\
\hline L1 & S1 & 161 & 172 & 11 \\
& S2 & 144 & 172 & 28 \\
L2 & S3 & 89 & 112 & 23 \\
& S4 & 89 & 112 & 23 \\
L3 & S5 & 33 & 93 & 60 \\
& S6 & 44 & 104 & 60 \\
\hline
\end{tabular}

\section{FABRICATION}

\subsection{CNC machining}

The aluminum parts of the EXOhSPEC collimator have been manufactured been manufactured by using NARIT's Hass VF-4 CNC machine. The material used is the 6061-T6 alloy material provided by a local manufacturer in Thailand. These parts are held in the machine with customized jig fixtures corresponding to each individual part. The lens barrel itself was the most delicate part. The dimension of concentric circles as well as the flatness of the surfaces has thus been carefully controlled during the manufacturing process. The retainers were also fabricated by using the same manufacturing method, and were tested with the barrel. These parts are shown in Figure 15 following black anodization.

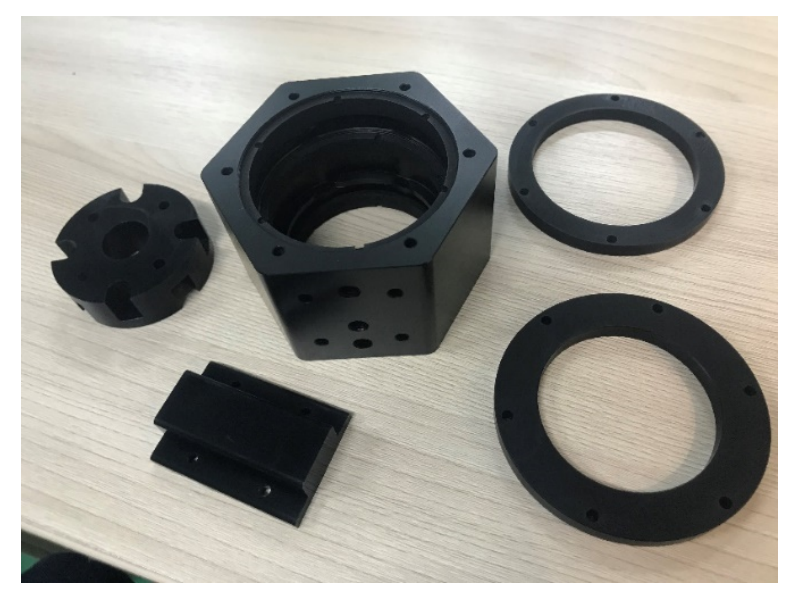

Figure 15. Aluminum parts fabricated at NARIT workshop

\subsection{CMM control}

We measured the dimensions of each referenced surface that will be used to position each lens at the correct location. These measurements have been done by using the Global Classic SR 05.07.05 CMM in an air conditioned temperaturecontrolled room. The results of the measurements of the L1, L2 and L3 internal diameters are shown in Table 7. We notice 
that the maximum deviation between the theoretical and the measured diameter is 19.5 microns and is obtained for the lens L3. For the lens L2 (respectively L3) the difference is 11.5 microns (respectively less than 1 microns).

We calculated from these measurements the maximum lateral XY-decenter of each lens as represented in Table 8 . We estimate that the L1 position error should be less than 1 microns that is smaller than the measurement accuracy $(2 \mu \mathrm{m})$ and totally negligible when compared to the mechanical specifications. The maximum position error of L2 (respectively of L3) should be 6 microns (respectively 10 microns) along X and 10 microns (respectively 17 microns) along Y. This is fully compliant to the specification of 25 microns and is better than the assumption used to establish EXOhSPEC performance budget [ref paper exohspec SPIE].

Table 7. Global Classic SR 05.07.05 CMM Measurement results measuring the deviation from the origin of concentric lateral references (based on a decenter).

\begin{tabular}{crrr}
\hline Lens & \multicolumn{2}{c}{ Theoretical } & Raw Measurement \\
Diameter $\emptyset_{\text {Ref }}(\mathrm{mm})$ & Diameter $\emptyset_{\text {Meas }}(\mathrm{mm})$ & $\begin{array}{c}\text { Error } \\
(\mu \mathrm{m})\end{array}$ \\
\hline L1 & 30.0000 & 30.0009 & $<1$ \\
L2 & 28.0000 & 27.9885 & 12 \\
L3 & 26.0000 & 26.0195 & 20 \\
\hline
\end{tabular}

Table 8. Maximum lateral XY-decenter calculated from the CMM measurement results.

\begin{tabular}{rrr}
\hline Lens & $\begin{array}{r}\text { Maximum } \\
\text { X-decenter }(\mu \mathrm{m})\end{array}$ & $\begin{array}{r}\text { Maximum } \\
\text { Y-decenter }(\mu \mathrm{m})\end{array}$ \\
\hline L1 & $<1$ & $<1$ \\
L2 & 6 & 10 \\
L3 & 10 & 17 \\
\hline
\end{tabular}

\section{CONCLUSION}

We present the mechanical design and performance analysis of the collimator for the EXOhSPEC achromatic triplet. This design is based on a semi-kinematic mounting for each lens using i) a geometrically controlled interface from the specification of the CNC machine, ii) an appropriate preload using three retainers with silicone elastomer in the axial direction, and iii) three ball-plungers to fix the positions of the lenses in the lateral plane.

The results of the Finite Element Analysis show that the maximum surface error over the optical surface covered by the incident beam and induced by the mounting is less than $60 \mathrm{~nm}$ PTV. The final system has the total weight of $716 \mathrm{~g}$ including the triplet. The collimator was fabricated at the NARIT high precision mechanical workshop and was controlled using the CMM. The results showed that mechanical performance are better that the specified tolerances with a maximum error of 17 microns on lens L3.

We thus conclude that the EXOhSPEC barrel was acceptable. The next steps of the collimator development will comprise four steps. First, we plan to assemble the lenses inside the barrel. Second, we will measure the collimator wavefront error by using a Shack-Hartman wavefront sensor in a double-pass configuration. Third, we will integrate the collimator into the spectrograph and we will measure the performance in laboratory conditions. Finally, we will measure the EXOhSPEC on-sky performance with the TNT between November 2019 and May 2020. 


\section{REFERENCES}

[1] E. Lhospice, C. Buisset, H. Jones, W. Martin, R. Errmann, S. Sithajan, C. Boonsri, P. Choochalerm, G. AngladaEscude, D. Campbell, M. A. Alagao, J.Paenoi, A. Prasit, T. Panyaphirawat, S. Rattanasoon, A.Leckngam, D. Mkrtichian, S.Aukkaravittayapun, "EXOhSPEC folded design optimization and performance estimation", Techniques and Instrumentation for Detection of Exoplanets IX (2019).

[2] C. Buisset; A. Prasit; A. Leckngam; T. Lépine; S. Poshyajinda; B. Soonthornthum; P. Irawati; A.Richichi; U. Sawangwit; V.Dhillon; L. K. Hard, "Progress on the prevention of stray light and diffraction effects on the Thai National Telescope", SPIE 9626, Optical Systems Design 2015: Optical Design and Engineering VI, 96262E (2015);

[3] Reference Focal Reducer + ICPS2017

[4] HAAS website (2019): https://www.haascnc.com/fr/machines/vertical-mills/vf-series/models/medium/vf-3.html

[5] Hexagon website (2019): https://www.hexagonmi.com/products/coordinate-measuring-machines/bridgecmms/global-classic

[6] Clifford, A. A. "Multivariate Error Analysis: A Handbook of Error Propagation and Calculation in ManyParameter Systems", John Wiley \& Sons, New York, (1973).

[7] Yoder Jr., P.R. and Vukobratovich, D. "Opto-Mechanical Systems Design”, 4th ed., CRC Press, Washington, (2008).

[8] Ahmad, A. "Handbook of Optomechanical Engineering", CRC Press, Washington, (2000).

[9] Hibbeler R.C. "Mechanics of materials", 9th ed., Pearson, New York, (2004).

[10] Hecht, E. "Optics", 4th ed., Pearson, San Francisco, (2002). 